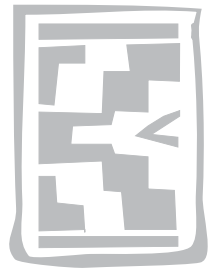

\title{
Age prevalence of trypanosomal infections in female Glossina morsitans morsitans (Diptera: Glossinidae) on the plateau area of eastern Zambia
}

\author{
C. KUBI ${ }^{1}$, M. BILLIOUW² and P. VAN DEN BOSSCHE ${ }^{1,3 *}$
}

\begin{abstract}
KUBI, C., VAN DEN BOSSCHE, P. \& BILLIOUW, M. 2007. Age prevalence of trypanosomal infections in female Glossina morsitans morsitans (Diptera: Glossinidae) on the plateau area of eastern Zambia. Onderstepoort Journal of Veterinary Research, 74:223-229

Trypanosomal infections in female Glossina morsitans morsitans were investigated in an area in the Eastern Province of Zambia between 1992 and 1994. A total of 4416 flies were captured, aged using the ovarian ageing method and screened for trypanosomal infections in both the mouthparts, salivary glands and the midgut. Congolense-type infections were identified in $4.8 \%$ of the flies. Vivax-type and immature infections were identified in $1.8 \%$ and $6.8 \%$ of the flies, respectively. The prevalence of congolense-type, vivax-type and immature infections increased with age. For vivax-type infections the age-prevalence relationship could be described by a model assuming a constant per capita rate of infection. For congolense-type and midgut infections, a polynomial term was added to the model significantly improving the fit. The per capita at which flies become infected was significantly higher for immature compared to mature infections. Observations strongly suggest that tsetse acquire new midgut infections at any age and that maturation of these infections is not limited to those obtained during the first blood meal.
\end{abstract}

Keywords: Age-prevalence, Glossina morsitans morsitans, Trypanosoma congolense, Trypanosoma vivax, Zambia

\section{INTRODUCTION}

The complex epidemiology of animal trypanosomosis is greatly affected by the number of infected tsetse transmitting the disease (Lambrecht 1980). Many ecological and physiological factors have been suggested to affect tsetse infection rates (Lambrecht 1980). Of particular epidemiological interest is the age prevalence of metacyclic trypanosomal infec-

* Author to whom correspondence is to be directed: E-mail: pvdbossche@itg.be

1 Department of Animal Health, Institute of Tropical Medicine, Nationalestraat 155, B-2000 Antwerp, Belgium

2 Thorite Road, 269 Garneton, Kitwe, Zambia

3 Department of Veterinary Tropical Diseases, Faculty of Veterinary Science, University of Pretoria, Private Bag X04, Onderstepoort, 0110 South Africa

Accepted for publication 19 March 2007-Editor tions in tsetse. This age-prevalence relationship has been described by various authors for different tsetse species (e.g. Harley 1966). Recently, a field study conducted in the Zambezi Valley (Zimbabwe) described this relationship for Glossina pallidipes using a mathematical model (Woolhouse, Hargrove \& McNamara 1993). At the same time, this model provided estimates of the developmental period of trypanosomes in tsetse and age-dependent susceptibility to infection.

This paper describes a study conducted in the Eastern Province, Zambia in which the age-prevalence relationship of trypanosomal infections in another tsetse species, Glossina morsitans morsitans was investigated. Whereas previous studies (Woolhouse et al. 1993; Woolhouse, Bealby, McNamara \& Silutongwe 1994) described this age-prevalence relationship for mature or metacyclic infections, this 
study gives an important additional dimension to the analysis by also investigating the age-prevalence relationship of immature or midgut infections. In an attempt to clarify the age-dependent maturation process of immature infections the fitted age-prevalence models of mature and immature infections are compared.

\section{MATERIAL AND METHODS}

\section{Study site}

The study was conducted between 1992 and 1994 in an area situated between $31^{\circ} 47^{\prime}-31^{\circ} 55^{\prime} \mathrm{E}$ and $13^{\circ} 55^{\prime}-14^{\circ} 12^{\prime} \mathrm{S}$ in Katete District, Eastern Province, Zambia. It is a highly cultivated area with a dense cattle population of about eight head of cattle per $\mathrm{km}^{2}$. Bovine trypanosomosis, transmitted by G. morsitans morsitans, is one of the major constraints for agricultural development.

\section{Tsetse sampling}

Tsetse were captured using man-walked fly rounds (Potts 1930) and F3-traps (Flint 1985). The odour bait used was acetone at a release rate of $250 \mathrm{mg} / \mathrm{h}$. Tsetse were sampled in different vegetation types (Brachystegia, riverine and Combretum woodland) during rainy, cold-dry and hot-dry seasons.

\section{Fly dissection}

Flies were dissected within $4 \mathrm{~h}$ after collection. Physiological age determination of females was conducted as described by Saunders (1960) and Challier (1965). Each fly was assigned to an ovarian age category $(\mathrm{OC}), 0-7$, depending on its ovarian configuration. Categories $0-1$ correspond to ages $0-8$ and 9-16 days, respectively and OCs $2-6$ correspond to additional intervals of 9 days, from 17-25 to 53-61 days, respectively. Depending on the content of the uterus, OCs 1-7 were subdivided into A (egg or first instar larva), B (second instar larva) or C (third instar larva). Ovarian category 0 was subdivided into A (immature egg) and B (mature egg). The categories were transformed into days corresponding to the pivotal age of each category.

Mouthparts, salivary glands and midgut dissections were performed using the method described by Lloyd \& Johnson (1924). Infections in the tsetse were identified according to the site of trypanosomal infestation. Infections in the proboscis alone were recorded as vivax-type, in the proboscis and the midgut as congolense-type and in the midgut alone as immature infections. The salivary glands were examined for mature brucei-type infections.

Throughout the analysis it was assumed that midgut or immature infections either mature into congolense-type infections or remain immature for the rest of the fly's life. Changes in the age-prevalence relationship of immature infections are thus due to either maturation of immature infections into congolensetype infections, acquisition of new midgut infections, or a combination of both. The age-prevalence relationship of the sum of immature and congolensetype infections, on the other hand, is only influenced by the development of new midgut infections. Consequently, the maturation of midgut infections or transmissibility was expressed as - congolensetype infections/immature infections + congolensetype infections (\%) per OAC.

\section{Statistical data analysis}

The raw fly data consist of three dichotomous response variables indicating, for each fly, the presence or absence of congolense-type, vivax-type and immature infections and a set of three explanatory variable, i.e. the year and month of fly capture and the ovarian age of the fly. The data are expressed in the form of prevalence of infection.

The first part of the analysis explored the effect of the three explanatory variables on the prevalence of the different infection types. It was assumed that the responses are from underlying binary distributions and consequently logistic regression was used to model the prevalences. Hypothesis testing was done by means of $\chi^{2}$-tests. The significance level was set at 0.01 . Since there was no significant interaction between the effects of age and time (year, month) for any of the infection types, the analysis of the ageprevalence curves proceeded using the pooled data.

The second part of the analysis estimated the per capita rate $(\lambda)$ at which flies become infected and the developmental period $(\tau)$ of the trypanosomes in the tsetse fly. Since logistic regression fits prevalences that are bounded between 0 and 1 , it was not appropriate for this purpose. Instead, we described the age-prevalence relationship for mature infections by the model used by Woolhouse et al. (1993):

$$
\begin{aligned}
& y(a)=1-\exp [-\lambda(a-\tau)] \text { or } \ln (1-y(a))=\lambda \tau-\lambda a \\
& \text { for } a>\tau ; y(a)=0 \text { for } a \leq \tau
\end{aligned}
$$

The age-prevalence relationship for immature infections was described using the following model: 


$$
\ln (1-y(a)-z(a))=\lambda ‘ a
$$

Where $y(a)$ is the proportion of infected flies at age $a$ ( $a$ is the pivotal age of each of the OCs) and $z(a)$ is the proportion of tsetse with immature infections at age a. The log-linear model was fitted to the ageprevalence data using least squares, i.e. with the assumption of approximate normality of In $(1-y)$ or In $(1-y-z)$. Polynomial terms were added when they significantly improved the model fit. The model's assumptions are discussed by Woolhouse (1989). The per capita rate at which tsetse become infected with immature infections ( $\lambda$ ') was compared with the per capita rate at which tsetse become infected with mature infections $(\lambda)$ using a t-test. The GLIM statistical software package was used for all statistical analyses.

\section{RESULTS}

Over the 2-year study period, 4416 female G. morsitans morsitans were sampled, screened for the presence of trypanosomal infections and aged using the ovarian dissection method. The annual totals were 2162 and 2254 flies for 1992 and 1993, respectively. Monthly sample sizes, pooled over both years, ranged from 175 in January to 599 in May.
Congolense-type infections, identified in 212 flies $(4.8 \%)$, were the dominant infection type. A total of $81(1.8 \%)$ flies had a vivax-type infection. The difference in prevalence is significant $\left(\chi^{2}=61, P<0.01\right)$. A total of $295(6.8 \%)$ flies had immature or midgut infections. One fly $(0.02 \%)$ had a brucei-type infection. Brucei-type infections were not included in the analysis.

\section{Temporal variation}

In 1992, the overall prevalence of trypanosomal infections was 277 flies out of a total of 2162 dissected flies (12.8\%). In 1993, a total of 311 flies out of 2254 dissected flies (13.8\%) were infected. The difference in prevalence between the 2 years was not significant $\left(\chi^{2}=0.93, P<0.01\right)$. Average monthly prevalence of trypanosomal infections ranged from $8 \%$ in June to $17.5 \%$ in July. This variation was significant $\left(\chi^{2}{ }_{11}=30, P<0.01\right)$. The shape of the monthly variation did not significantly differ between both years (month.year interaction: $\chi^{2}{ }_{11}=19, P<0.01$ ).

The effects of year and month on the prevalences, allowing for the effect of the age structure of the flies, were explored for congolense-type, vivax-type and the sum of immature and congolense-type infections. Results are presented in Table 1.

TABLE 1 Logistic regression analysis of deviance of terms affecting the prevalence of congolense-type, vivax-type and immature + congolense-type infections in female G. morsitans morsitans

\begin{tabular}{|c|c|c|c|c|}
\hline Terms included & Terms added & $\chi^{2}$-value & d.f. & $P$-value \\
\hline \multicolumn{5}{|c|}{ Congolense-type infections } \\
\hline $\begin{array}{l}- \\
- \\
- \\
\text { Age } \\
\text { Age }\end{array}$ & $\begin{array}{l}\text { Year } \\
\text { Month } \\
\text { Age } \\
\text { Year } \\
\text { Month }\end{array}$ & $\begin{array}{r}3.2 \\
15.5 \\
87.1 \\
3.3 \\
18.1\end{array}$ & $\begin{array}{r}1 \\
11 \\
1 \\
1 \\
11\end{array}$ & $\begin{aligned} & 0.07 \\
& 0.16 \\
< & 0.001^{\mathrm{a}} \\
& 0.07 \\
& 0.07\end{aligned}$ \\
\hline \multicolumn{5}{|c|}{ Vivax-type infections } \\
\hline $\begin{array}{l}- \\
- \\
\text { Month } \\
- \\
\text { Age } \\
\text { Age } \\
\text { Age + month }\end{array}$ & $\begin{array}{l}\text { Year } \\
\text { Month } \\
\text { Month.year } \\
\text { Age } \\
\text { Year } \\
\text { Month } \\
\text { Age.month }\end{array}$ & $\begin{array}{r}3.5 \\
25.7 \\
13.9 \\
14.2 \\
3.5 \\
25.0 \\
11.9\end{array}$ & $\begin{array}{r}1 \\
11 \\
12 \\
1 \\
1 \\
11 \\
11\end{array}$ & $\begin{aligned} & 0.06 \\
& 0.007^{\mathrm{a}} \\
& 0.30 \\
< & 0.001^{\mathrm{a}} \\
& 0.06 \\
& 0.009^{\mathrm{a}} \\
& 0.37\end{aligned}$ \\
\hline \multicolumn{5}{|c|}{ Immature + congolense-type infections } \\
\hline $\begin{array}{l}- \\
- \\
\text { Month } \\
- \\
\text { Age } \\
\text { Age } \\
\text { Age + month }\end{array}$ & $\begin{array}{l}\text { Year } \\
\text { Month } \\
\text { Month.yearb } \\
\text { Age } \\
\text { Year } \\
\text { Month } \\
\text { Age.month }\end{array}$ & $\begin{array}{r}1.6 \\
39.9 \\
18.3 \\
19.5 \\
31.7 \\
36.4 \\
6.9\end{array}$ & $\begin{array}{r}1 \\
11 \\
12 \\
1 \\
1 \\
11 \\
11\end{array}$ & $\begin{aligned} & 0.20 \\
< & 0.001^{\mathrm{a}} \\
& 0.10 \\
< & 0.001^{\mathrm{a}} \\
& 0.19 \\
< & 0.001^{\mathrm{a}} \\
& 0.8\end{aligned}$ \\
\hline
\end{tabular}



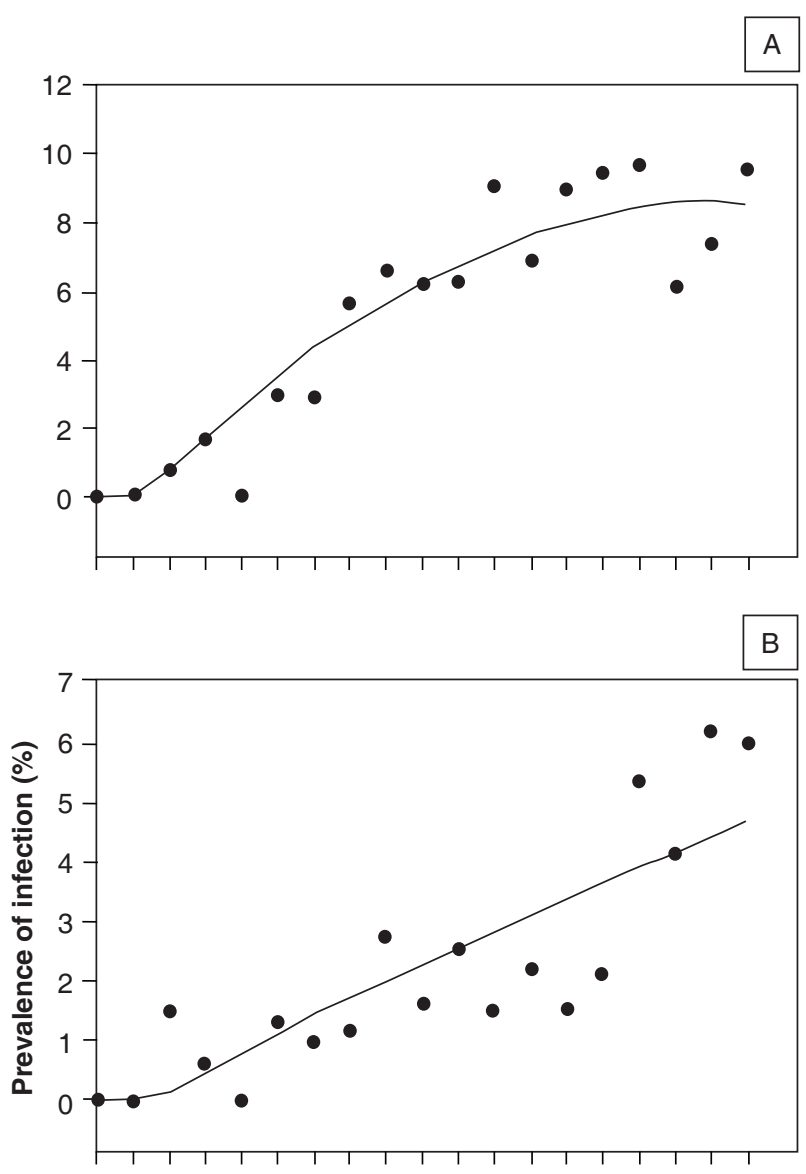

C

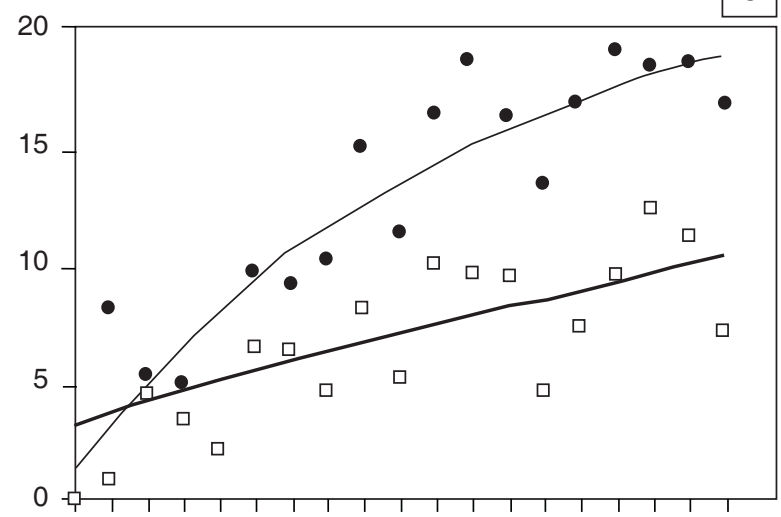

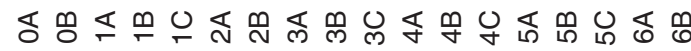

\section{Ovarian category}

FIG. 1 Variations in prevalence of trypanosomal infections in female Glossina morsitans morsitans with fly age (by ovarian category)

A Congolense-type infection. The best fit model (line) is $\ln (1-y)=0.02107-0.002672$ age +2.297 e-07 age $^{3}$

B Vivax-type infection. The best fit model (line) is In (1 $-\mathrm{y})=0.007950-0.0008496$ age

C Congolense-type infection $(\bullet)+$ immature. The best fit model (line) is $\ln (1-y)=-0.004448$ age + 0.00000032 age $^{3}$ and immature $(\square)$ only
TABLE 2 Parameter estimates of age-prevalence models for three trypanosomal infection-types in female G. morsitans morsitans

\begin{tabular}{|c|c|c|}
\hline Estimate & \pm S.E. & Parameter \\
\hline \multicolumn{3}{|c|}{ Congolense-type } \\
\hline $\begin{array}{l}-0.02107 \\
-0.002672 \\
2.292 \text { e-07 }\end{array}$ & $\begin{array}{l}0.009337 \\
0.0004328 \\
9.391 \text { e- } 08\end{array}$ & $\begin{array}{l}1 \\
\text { Age } \\
\text { Age }^{3}\end{array}$ \\
\hline \multicolumn{3}{|l|}{ vivax-type } \\
\hline $\begin{array}{c}0.007950 \\
-0.0008496\end{array}$ & $\begin{array}{l}0.005302 \\
0.0001323\end{array}$ & $\begin{array}{l}1 \\
\text { Age }\end{array}$ \\
\hline \multicolumn{3}{|c|}{ Immature + congolense-type } \\
\hline $\begin{array}{l}-0.00448 \\
0.00000032\end{array}$ & $\begin{array}{l}0.0003929 \\
0.00000012\end{array}$ & $\begin{array}{l}\text { Age } \\
\mathrm{Age}^{3}\end{array}$ \\
\hline
\end{tabular}

There was no significant yearly variation in the prevalences of any of the infection types and no effect of year on the shape of the monthly variation of any of the infection types. For congolense-type infections, only age had a significant effect on prevalence. Monthly variation in prevalences were not significant. For vivax-type infections both age and month, but not their interaction, were significant factors. The monthly prevalence of vivax-type infections varied from $0.7 \%$ in October to $3.6 \%$ in December. For the sum of immature and congolense-type infections both age and month, but not their interaction, were significant factors explaining the variation in the prevalence. Average estimates of infection prevalences with age, for the whole of the study period, were obtained, ignoring the effects of month and year.

\section{Age-prevalence relationship}

The observed age-prevalence relationship for congolense-type and vivax-type infections are shown in Fig. $1 A$ and $B$.

The prevalence of congolense-type infections ranged from $0 \%$ (OCs $0 A$ and $0 \mathrm{~B}$ ) to $9.5 \%$ (OC 6B). For vivax-type infections the prevalence varied between $0 \%(\mathrm{OC} 0 \mathrm{~A})$ to $6.1 \%(\mathrm{OC} 6 \mathrm{~B})$. The model In $(1-y)=\lambda \tau-\lambda a$ was fitted to the age prevalence data of the congolense-type and vivax-type infections to obtain estimates of the per capita infection rate $(\lambda)$ and developmental period $(\tau)$. The fitted curves are listed in Table 2 and plotted, together with the observed prevalences, in Fig. $1 \mathrm{~A}$ and $\mathrm{B}$.

For the congolense-type infections, the model included a cubic term of age which significantly improved the fit $\left(F_{1,16}=5.95, P<0.01\right)$. The model 


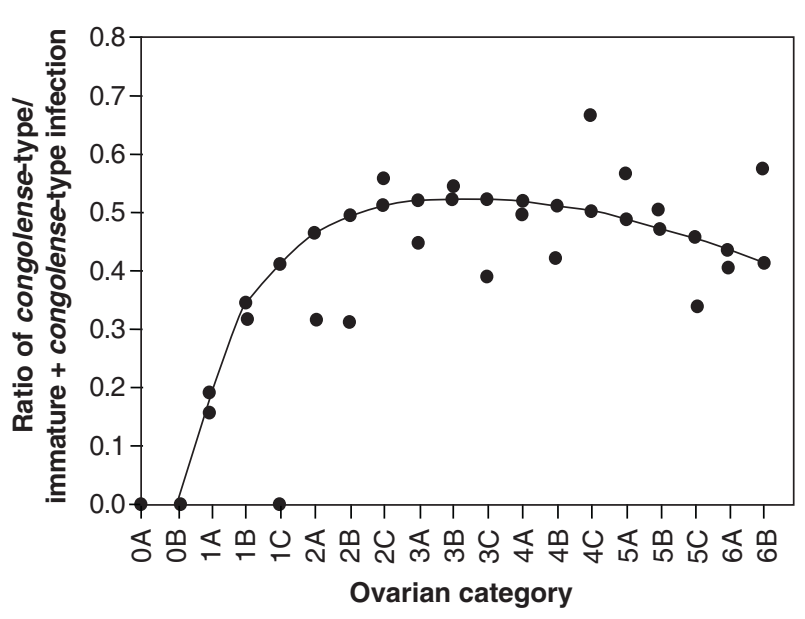

FIG. 2 Variations in the ratio of congolense-type infections/ congolense-type + immature infections (ם) of female Glossina morsitans morsitans with fly age (by ovarian category)

explained $87 \%$ and $71 \%$ of the variation in $\ln (1-y)$ for congolense-type and vivax-type infections, respectively. The parameter estimates $( \pm$ standard error) for congolense-type infections were $\tau=7.9 \pm$ 3.7 days and $\lambda=0.00261 \pm 0.00043$ per fly per day at age $=\tau$. For vivax-type infections, the parameter estimates ( \pm standard error) were $\tau=9.4 \pm 6.5$ days and $\lambda=0.00085 \pm 0.00013$ per fly per day at age $=\tau$.

The prevalence of immature infections varied between $0 \%(\mathrm{OC} O A)$ to a maximum of $12.2 \%$ (OC $5 \mathrm{C})$. The observed prevalences for immature only and the sum of immature and congolense-type infections are presented in Fig. 1C.

The model for immature + congolense-type infections was fitted without intercept $\left(F_{1,16}=0.01, P<\right.$ $0.05)$. A cubic term significantly improved the fit $\left(F_{1.17}=6.1, P<0.05\right)$. This model explains $91.5 \%$ of the variation of the prevalence of immature + congolense-type infections. The fitted curve is plotted together with the observed prevalences in Fig. 1C. The parameter estimates of the fitted model are given in Table 2 . The per capita rate $(\lambda)$ at which flies become infected was estimated at $0.00447 \pm$ 0.00039 per fly per day at Day 0 . The estimate of $\lambda^{4}$ at age $\tau$ for mature infections is significantly lower $\left(\mathrm{t}_{33}=2.958 ; P=0.0028\right)$ than the estimate of $\lambda$ for immature infections at Age 0.

The observed and fitted ratio of congolense-type infections/immature infections + congolense-type infections, for each of the age groups, is plotted in Fig. 2. The ratio increases rapidly to a value of about 0.5 at the age of about 30 days after which it remains almost constant.

\section{DISCUSSION}

Except for studies conducted by Clarke (1969) and Woolhouse et al. (1994) little information is available on the prevalence of trypanosomal infections in Zambian tsetse species. Contrary to Clarke's observations, congolense-type infections are the most prevalent in our study area. This is in accordance with the high bovine trypanosomosis prevalence, caused by $T$. congolense, in the plateau area of the Eastern Province.

The per capita rate at which female G. morsitans morsitans acquire congolense-type infections, suggests that $0.26 \%$ of the flies become infected per day. At a feeding interval of 4 days (Rogers 1988) and a $75 \%$ feeding preference for cattle (as is the case in the study area), this corresponds to a successful infection every 72 blood meals on cattle. The prevalence of $T$. congolense infections in cattle is high in the study area. At a prevalence of $40 \%$, $3.5 \%$ of the feeds on infected cattle develop and mature in female $G$. morsitans morsitans. This figure is slightly higher than the $2.5 \%$ obtained by Rogers \& Boreham (1973) from an analysis of data on $G$. swynnertoni in Tanzania.

The estimated developmental period of congolensetype infections in G. morsitans morsitans corresponds well with the average 8-9 days observed by Elce (1971). Nantulya, Doyle \& Jenni (1978), however, observed development periods up to 40 days. The estimated developmental period for vivax-type infections agrees with the 5-13 days reported by Davies (1977).

Results from this study show that the prevalence of vivax-type infections rises approximately linearly with fly age. This is what could be expected and reinforces the observations that tsetse flies can readily infect themselves with $T$. vivax throughout their life. This is most likely related to the relatively simple developmental cycle of $T$. vivax in the tsetse fly.

The age-prevalence curve of immature + congolense-type infections strongly suggests that, in accordance with laboratory observations (Welburn \& Maudlin 1992), wild tsetse also acquire new trypanosomal midgut infections at any age. Consequently, infected blood meals can give rise to immature infections even when these blood meals are taken by non-teneral flies.

There is some conflicting evidence concerning the ease with which non-teneral tsetse flies can infect themselves with $T$. congolense. Many authors, using both laboratory-reared tsetse and tsetse emerging 
from wild pupae, have described the significantly higher susceptibility of teneral compared to non-teneral flies to T. congolense infections (Owaga 1981; Distelmans, D'haeseleer, Kaufman \& Rousseeuw 1982; Mwangelwa, Otieno \& Reid 1987; Welburn \& Maudlin 1992; Dale, Welburn, Maudlin \& Milligan 1995). On the other hand, Harley (1967) found an increasing $T$. congolense-infection prevalence with increasing age in females of several tsetse species. It is nevertheless generally accepted that tsetse, once fed, are relatively but not completely refractory to trypanosomal infections. Consequently, infections acquired by non-teneral wild flies are expected to play a minor role in the epidemiology of tsetsetransmitted trypanosomosis.

According to our observations, the prevalence of congolense-type infections increases substantially, though not linearly, with increasing age. The fit of the model to the age-prevalence data was improved by an additional cubic term. This age-prevalence model can be explained by variations in incubation time of infections obtained in young flies, age-dependent decrease in susceptibility to infection or increased mortality of tsetse infected with a metacyclic T. congolense infection (Dale et al. 1995; Woolhouse et al. 1993).

The developmental or incubation period of congolense-type infections can vary (Dale et al. 1995). Midgut or immature infections, however, must develop immediately after the ingestion of the infected blood meal. Assuming that maturation is restricted to infections obtained during the first blood meal, the prevalence of midgut infections in the youngest age categories cannot be lower than the maximum prevalence of mature, congolense-type infections in the subsequent age categories. This could not be confirmed by our observations. The maximum congolense-type infection prevalence $(8.6 \%$ in OC 6$)$ is higher than the maximum midgut-infection prevalence in the first three age categories (6\%). Furthermore, our results show that between OCs 2 and 6 the congolense-type infection prevalence almost doubles from $4.5 \%$ to $8.6 \%$. This increase in congolense-type infections cannot be due to retarded maturation of trypanosomal infections obtained during the first blood meal, since the prevalence of immature infections in the first age categories only varies between $0 \%$ and $4 \%$. Consequently, the increase in the congolense-type infection prevalence with increasing age is a result of maturation of newly acquired midgut infections.

Moreover, if few of the newly acquired midgut infections in non-teneral tsetse would mature, one would expect a decreasing ratio of congolense-type infections/immature + congolense-type infections once the developmental period of the infections acquired during the first blood meal is over. This is not the case.

Although our results indicate that maturation of trypanosomal congolense-type infections is not restricted to those obtained during the first blood meal, the shape of the ratio of congolense-type infections/ immature infections + congolense-type infections curve suggests that trypanosomal infections obtained early in life contribute more to the congolensetype prevalence than those acquired at a later age. Moreover, there is a significant difference between the force-of-infection for immature infections $\left(\lambda^{\prime}\right)$ and the force-of-infection for mature congolense-type infections $(\lambda)$. This implies that immature infections do not all mature to become mature infections some weeks later. There are two obvious explanations: either there is an increased mortality of immature infected flies, or there is some recovery from infections. Welburn, Maudlin \& Ellis (1989) showed that, under laboratory conditions, it can take up to 7 days to remove an incoming infection from the midgut of a tsetse fly. Consequently, some of these "non-permanent" midgut infections will be detected and classified as midgut infection for a particular age group but will be removed from the midgut in a higher age group.

Observations described in this paper confirm the age-prevalence relationship of trypanosomal infections in wild tsetse reported in other recent studies and another tsetse species (Woolhouse et al. 1993; Woolhouse et al. 1994). Moreover, our results clearly indicate that the increase in the prevalence of congolense-type infections with increasing age is due to the maturation of midgut infection acquired by non-teneral tsetse flies. Contrary to laboratory observations (Welburn \& Maudlin 1992), these newly acquired infections substantially contribute to the overall congolense-type infection rate of the tsetse population.

\section{ACKNOWLEDGEMENTS}

The authors are grateful to the staff of the Chipopela Research Station where the work was conducted. Drs M.E.J. Woolhouse and R. de Deken are kindly acknowledged for their comments on the manuscript. The work was funded by the Belgian Development Co-operation through the Assistance to the Veterinary Services of Zambia project. 


\section{REFERENCES}

CHALLIER, A. 1965. Amélioration de la méthode de détermination de l'âge physiologique des Glossines. Etudes faites sur Glossina palpalis gambiensis Vanderplank, 1949. Bulletin de la Société Pathology Exotique, 58:250-259.

CLARKE, J.E. 1969. Trypanosome infection rates in the mouthparts of Zambian tsetse flies. Annals of Tropical Medicine and Parasitology, 63:15-34.

DALE, C., WELBURN, S.C., MAUDLIN, I. \& MILLIGAN, P.J.M. 1995. The kinetics of maturation of trypanosome infections in tsetse. Parasitology, 111:187-191.

DAVIES, H. 1977. Tsetse flies in Nigeria, $3^{\text {rd }}$ ed. Ibadan: Oxford University Press.

DISTELMANS, W., D'HAESELEER, F., KAUFMAN, L. \& ROUSSEEUW, P. 1982. The susceptibility of Glossina palpalis palpalis at different ages to infection with Trypanosoma congolense. Annales de la Société Belge Médecine Tropicale, 62:41-47.

ELCE, B.J. 1971. The transmission of Trypanosoma congolense through Glossina morsitans and the white mouse. Transactions of the Royal Society of Tropical Medicine and Hygiene, 65:239.

FLINT, S. 1985. A comparison of various traps for Glossina spp. (Glossinidae) and other Diptera. Bulletin of Entomologic Research, 75:529-534.

HARLEY, J.M.B. 1966. Studies on age and infection rate of Glossina pallidipes Austen, G. palpalis_fuscipes and G. brevipalpis Newst. in Uganda. Bulletin of Entomologic Research, 57:23-37.

HARLEY, J.M.B. 1967. Further studies on age and trypanosome infection rate in Glossina pallidipes Aust., G. palpalis fuscipes and G. brevipalpis Newst. in Uganda. Bulletin of Entomologic Research, 5:459-477.

LAMBRECHT, F.L. 1980. Ecological and physiological factors in the cyclic transmission of African Trypanosomiasis. Insect Science and its Application, 1:47-54.

LLOYD, L.L. \& JOHNSON, W.B. 1924. The trypanosome infections of tsetse flies in Northern Nigeria and a method of estimation. Bulletin of Entomologic Research, 14:25-257.

MWANGELWA, M.I., OTIENO, L.H. \& REID, G.D.F. 1987. Some barriers to Trypanosoma congolense development in Glos- sina morsitans morsitans. Insect Science and its Application, 1:33-37.

NANTULYA, V.M., DOYLE, J.J. \& JENNI, L. 1978. Studies on Trypansoma_(Nannomonas)_congolense 2. Observations on the cyclical transmission of three field isolates by Glossina morsitans morsitans. Acta Tropica, 35:339-344.

OWAGA, M.L.A. 1981. Trypanosome infection rate in the tsetse species; Glossina pallidipes Austen, in a rural situation in Kenya. Insect Science and its Application, 1:411-416.

POTTS, W.H. 1930. A contribution to the study of numbers of tsetse-fly (Glossina morsitans Westw.) by quantitative methods. South African Journal of Science, 27:491-497.

ROGERS, D.J. 1988. A general model for the African trypanosomiasis. Parasitology, 97:193-212.

ROGERS, D.J. \& BOREHAM, P.F.L. 1973. Sleeping sickness survey in the Serengeti area (Tanzania) 1971 II. The vector role of Glossina swynnertoni Austen. Acta Tropica. 30:2435.

SAUNDERS, D.S. 1960. The ovulation cycle in Glossina morsitans Westwood (Diptera: Muscidae) and a possible method of age determination for female tsetse flies by examination of their ovaries. Transactions of the Royal Society of Tropical Medicine and Hygiene, 112:221-238.

WELBURN, S.C., MAUDLIN, I. \& ELLIS, D.S. 1989. Rate of trypanosome killing by lectins in midguts of different species and strains of Glossina. Medical and Veterinary Entomology, 3:77-82.

WELBURN, S.C. \& MAUDLIN, I. 1992. The nature of the teneral state in Glossina and its role in the acquisition of trypanosome infection in tsetse. Annals of Tropical Medicine and Parasitology, 86:529-536.

WOOLHOUSE, M.E.J. 1989. On the interpretation of age-prevalence curves for schistosome infections of host snails. Parasitology, 99:47-56.

WOOLHOUSE, M.E.J., HARGROVE, J.W. \& MCNAMARA, J.J. 1993. Epidemiology of trypanosome infections of the tsetse fly Glossina pallidipes in the Zambezi Valley. Parasitology, 106:479-485.

WOOLHOUSE, M.E.J., BEALBY, K., MCNAMARA, J.J. \& SILUTONGWE, J. 1994. Trypanosome infections of the tsetse fly Glossina pallidipes in the Luangwa Valley, Zambia. International Journal of Parasitology, 24:987-993. 